\title{
Diagnostic techniques for measuring suprathermal electron dynamics in plasmas (invited) ${ }^{a}$
}

\author{
S. Coda ${ }^{\text {) }}$ \\ Ecole Polytechnique Fédérale de Lausanne (EPFL), Centre de Recherches en Physique des Plasmas \\ Association Euratom-Confédération Suisse, EPFL SB CRPP, Station 13, CH-1015 Lausanne, Switzerland
}

(Presented 15 May 2008; received 12 May 2008; accepted 14 July 2008; published online 31 October 2008)

\begin{abstract}
Plasmas, both in the laboratory and in space, are often not in thermodynamic equilibrium, and the plasma electron distribution function is accordingly non-Maxwellian. Suprathermal electron tails can be generated by external drives, such as rf waves and electric fields, or internal ones, such as instabilities and magnetic reconnection. The variety and importance of the phenomena in which suprathermal electrons play a significant role explains an enduring interest in diagnostic techniques to investigate their properties and dynamics. X-ray bremsstrahlung emission has been studied in hot magnetized plasmas for well over two decades, flanked progressively by electron-cyclotron emission in geometries favoring the high-energy end of the distribution function (high-field-side, vertical, oblique emission), by electron-cyclotron absorption, by spectroscopic techniques, and at lower temperatures, by Langmuir probes and electrostatic analyzers. Continuous progress in detector technology and in measurement and analysis techniques, increasingly sophisticated layouts (multichannel and tomographic systems, imaging geometries), and highly controlled suprathermal generation methods (e.g., perturbative rf modulation) have all been brought to bear in recent years on an increasingly detailed, although far from complete, understanding of suprathermal electron dynamics. (C) 2008 American Institute of Physics. [DOI: 10.1063/1.2966599]
\end{abstract}

\section{INTRODUCTION}

A plasma at ideal thermodynamic equilibrium is described by the Maxwell-Boltzmann kinetic distribution function. Plasmas are, however, complex systems to which this ideal seldom applies. In the laboratory, high-temperature plasmas of thermonuclear interest are confined preferentially by magnetic fields or by their own inertia, in dynamical configurations that are intrinsically spatially nonuniform. Thermodynamic quantities can, therefore, only be defined locally, while free energy remains stored in their spatial gradients. Moreover, present-day experiments are strongly driven systems, subject to electric fields, varying magnetic fields, rf waves, laser beams, neutral beams, ion beams, etc., all of which can act to render even a local Maxwellian description inadequate. Even in the most quiescent cases, such as lowtemperature laboratory setups or some instances of space plasmas, the complexity inherent in a medium composed of charged particles ensures that collective motions and modes can always be present, keeping the system away from true equilibrium.

To first order, a departure from equilibrium results in the appearance of a suprathermal population superimposed on an approximately Maxwellian bulk. The suprathermal population is often the direct result of the interaction with the various external drives; accordingly, its properties and dynamical

\footnotetext{
a) Invited paper, published as part of the Proceedings of the 17th Topical Conference on High-Temperature Plasma Diagnostics, Albuquerque, New Mexico, May 2008

${ }^{b)}$ Electronic mail: stefano.coda@epfl.ch.
}

evolution are an integral component of the description of the application and effects of those drives, and are generally important in evaluating the results of a particular experimental scenario. Such is the case of heating by rf waves in fusion devices and more particularly of the rf current-drive mechanism, which depends upon generating an asymmetric electron distribution function (e.d.f.) in velocity space. Electric fields, which are applied to drive currents in toroidal devices, also result in deformations of the e.d.f.. In laser fusion, suprathermal electrons can be generated by the resonant absorption mechanism and can result in target preheating, a deleterious effect limiting compression and potentially preventing ignition. In magnetized plasmas, electric fields accompany the phenomenon of magnetic reconnection, and the resulting acceleration produces suprathermal tails that are widely observed in the solar corona, in the aurora region, and in laboratory plasmas-typically in connection with the formation of magnetic islands such as in sawtooth and disruptive instabilities in tokamaks.

A growing realization of the importance of the highenergy end of the e.d.f. in plasmas has accompanied the progress of experimental plasma physics over the past four decades and has spurred the development of increasingly sophisticated diagnostic techniques to investigate the suprathermal electron population. Hard x-rays (HXRs) have historically been the most obvious indicator of the presence of such a population and accordingly resulted in the first dedicated suprathermal electron diagnostics for high-temperature plasmas, as well as in standard solar diagnostics. Steady technological developments, particularly in solid-state detectors 
progressively replacing the unwieldy early apparatus, have permitted this technique to evolve into a mature discipline, with high resolution both in space and energy being the norm nowadays, and many-chord tomographic systems starting to appear. The high photon energy translates to poor statistics, an unavoidable obstacle to good temporal resolution in spite of the intrinsically fast detectors available.

A way around the latter hurdle is offered, in magnetized plasmas, by electron-cyclotron radiation, which involves far lower photon energies and much higher fluences in most cases of interest. Electron-cyclotron emission (ECE) has historically been associated with localized electron temperature measurements because of the generally large optical depth of fusion plasmas, resulting in easily analyzable blackbody emission. The presence of suprathermal electrons causes deviations from the blackbody ideal, and these deviations can therefore be used to diagnose the suprathermal population. While technological development has naturally played a role in this discipline too, credit must be given to the considerable ingenuity invested in devising experimental setups and geometrical arrangements to maximally constrain the interpretation of the results. Even though ECE-based methods suffer from an intrinsic convolution of energy and resonance location, it has proven possible in many cases to deconvolve the information at least partly.

Methods based on atomic spectroscopy have also been developed in the past decade to provide some information on e.d.f. anisotropy. These techniques are not yet widespread in high-temperature plasmas but may well grow in the near future. Other direct or indirect measurement techniques have also been applied to the problem of nonthermal electron distributions, including Langmuir probes, pyrobolometers and optical pyrometers, radio-wave emission, and in situ electron spectrometers. These techniques are somewhat marginal to the diagnosis of high-temperature plasmas as they rely specifically on partial ionization or low temperature. However, as a high-temperature confined plasma will always have a low-temperature edge, such diagnostics remain valuable in a variety of practical situations.

This paper does not aim to be a general review of the subject. Rather, it aims to provide a general overview, heavily weighted toward magnetic-confinement fusion. It is hoped, however, that the reader interested in applications in the broader realm of plasma measurements will find the references and citations provided a useful guide for pursuing this interest further. A historical perspective is privileged, with a view to chart the progress of the techniques in concert with the scientific motivations on one hand and the technological developments on the other.

The remainder of this paper is organized as follows. In Secs. II and III the primary diagnostic techniques based on hard x-rays and electron-cyclotron radiation, respectively, will be reviewed and discussed. Section IV will then provide an overview of the remaining techniques.

\section{HARD-X-RAY BREMSSTRAHLUNG}

High-energy electrons emit continuum bremsstrahlung radiation $^{1,2}$ upon undergoing Coulomb collisions with ions

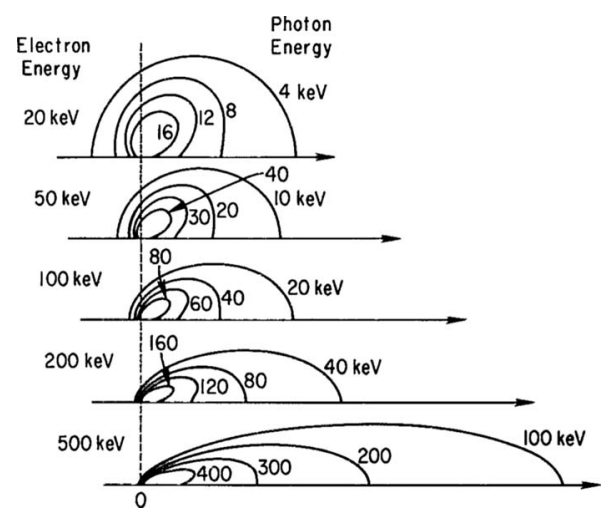

FIG. 1. Bremsstrahlung emission cones for electrons of different energies. Electrons travel from left to right. The length of a vector from the origin to a point on a curve is proportional to the bremsstrahlung power emitted in that direction at the photon energy associated with that particular curve. Reprinted with permission from von Goeler et al. (Ref. 23).

and to a considerably lesser extent, with electrons of unlike energy (symmetry considerations dictate that two colliding electrons of equal relativistic mass generate radiation fields that cancel exactly). Thick-target bremsstrahlung is also emitted by electrons escaping the plasma and coming to rest in a solid target. Depending on the impact parameter, an electron can emit photons with energy up to its initial energy. In a keV-grade plasma, suprathermal electron bremsstrahlung therefore tends to be predominantly in the HXR region of the spectrum. Head-on collisions result in higher-energy radiation, preferentially aligned with the initial electron velocity, the anisotropy being enhanced at high electron energies by the relativistic headlight effect (see Fig. 1).

In magnetic-confinement fusion the early development of HXR diagnostics ${ }^{3}$ was driven by investigations of e.d.f. asymmetries in mirror machines, ${ }^{4}$ as well as runaway electrons and lower-hybrid current drive (LHCD), particularly in the pioneering work of von Goeler and co-workers on the $\mathrm{ST}^{3,5}$ Princeton large torus (PLT), ${ }^{6}$ and Princeton beta experiment-modification $(\mathrm{PBX}-\mathrm{M})^{7}$ devices at the Princeton Plasma Physics Laboratory. The LHCD mechanism was one of the earliest ${ }^{8,9}$ and to this day, the most efficient means of driving a noninductive electric current in a plasma, and is based on an asymmetric e.d.f. distortion in the velocity component parallel to the magnetic field, both through direct parallel momentum transfer and through the generation of an asymmetric resistivity. ${ }^{10}$ The resulting e.d.f. is calculated to have a broad plateau in the forward parallel direction. It was therefore paramount to diagnose the distorted e.d.f. and the current-carrying high-parallel-velocity electrons. Tangentially viewing lines of sight were accordingly privileged, although angular scanning was soon made possible in order to directly ascertain and confirm the expected asymmetry. The angular distribution was indeed found to be peaked in the forward direction.

The basic dynamics of the establishment of the suprathermal tail were rapidly determined, but the next level of investigation, i.e., characterizing the form of the e.d.f., presented a bigger challenge. A fundamental limitation of bremsstrahlung-based measurements is that the electron energy distribution cannot be derived from the photon energy 

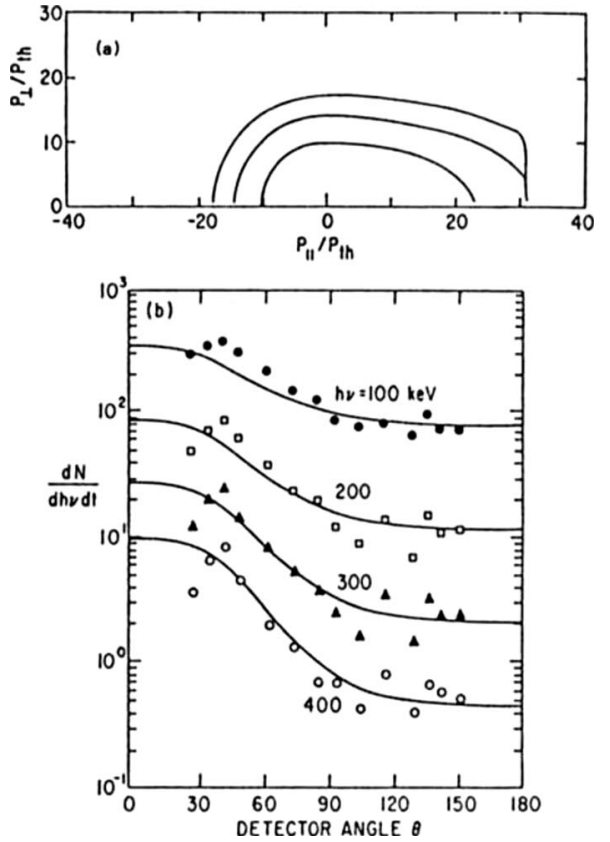

FIG. 2. (a) Contours of a model suprathermal distribution function plotted vs parallel and perpendicular momentum (normalized to the thermal momentum of a $1 \mathrm{keV}$ temperature bulk plasma). (b) Photon counts vs detector viewing angle relative to the magnetic field: measurements on the PLT tokamak (symbols) and fits from model distribution (curves). Reprinted with permission from Stevens et al. (Ref. 11).

distribution in general: the problem is ill posed in that no unique solution exists. The Princeton team took a practical, partly model-based approach, in which the e.d.f. was parametrized as an anisotropic multi-Maxwellian function with a perpendicular, forward parallel, and backward parallel temperatures, in addition to a maximum momentum. The four free parameters could then be determined by a fit to the HXR measurements, ${ }^{11}$ as shown in Fig. 2. This approach was proven robust and successful, was later adopted on a variety of devices employing LHCD, ${ }^{12-17}$ and was also improved computationally by being incorporated in a ray-tracing code. ${ }^{18}$ An inversion technique was proposed in a different study to reconstruct a global anisotropy coefficient. ${ }^{19}$

These early measurements were based on scintillator technology. Materials such as $\mathrm{NaI}^{6,12,14,15,20,21} \mathrm{CsI}^{7,16}$ and $\mathrm{BGO}^{22}$ were used in scintillators coupled to photomultipliers and image intensifiers ${ }^{7,23}$ (see Fig. 3). Foil techniques were also used for energy selectivity. ${ }^{24}$ These detectors were large and had the additional need for bulky cooling and magneticshielding materials. Nevertheless, the reliability of the technology is such that it is still in use today. Fast scintillators are also employed on laser-fusion experiments to detect HXR emitted by suprathermal electrons generated by the laserplasma instabilities, such as two-plasmon decays and stimulated Raman scattering. ${ }^{25,26}$ These fast electrons are responsible for target preheating, which can reduce compression and inhibit ignition in both indirect- and direct-drive inertial fusion. HXR measurements have been proven instrumental in diagnosing the occurrence of these events. Plastic scintillators coupled with microchannel-plate photomultipliers are employed, both possessing subnanosecond time response,

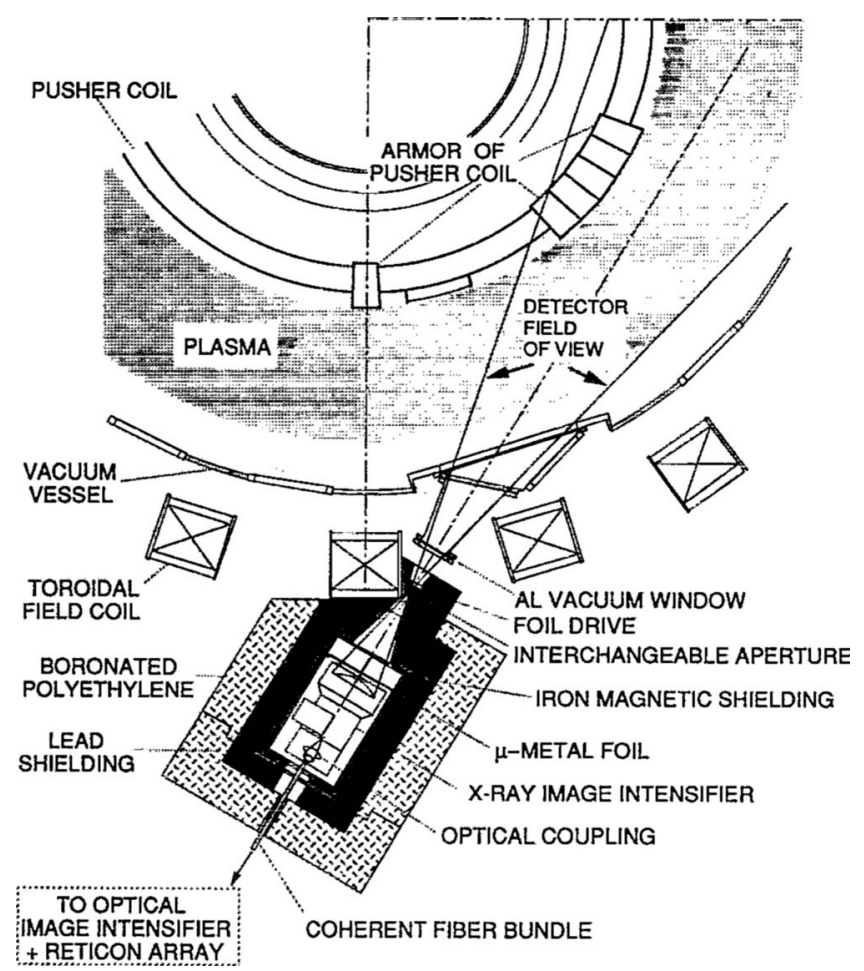

FIG. 3. Mechanical layout of a HXR pinhole camera on the PBX-M tokamak, with iron magnetic shielding and lead and borated-polyethylene neutron and $\gamma$-ray shielding. Reprinted with permission from von Goeler et al. (Ref. 23).

permitting a crucial separation of the early HXR generated by suprathermals from the radiation emitted at compression time. $^{26}$

Thick-target $K \alpha$ radiation from escaping suprathermal electrons can also be detected with crystal spectrometers, which have been widely employed as a diagnostic in laser fusion. ${ }^{27}$ In cases with significant fractions of suprathermal electrons escaping fusion plasmas, thick-target bremsstrahlung has also been proven useful in providing insight into their distribution. ${ }^{28-33}$

A key question on the generation of current-carrying suprathermal electrons concerns their confinement within the plasma column. The suprathermal population experiences a natural sink due to slowing-down from collisions. Any additional dissipation mechanisms, such as cross-field transport, would act to reduce the efficiency of sustaining a suprathermal tail and to broaden the noninductive current profile. ${ }^{34}$ Studies of the dynamics of suprathermal electrons have accordingly accompanied the entire history of HXR measurements, particularly with LHCD, to test the oft made assumption that the HXR emission profile is proportional to the driven current-density profile. ${ }^{3}$ A particular concern is magnetic turbulence. While the level of magnetic turbulence, $\widetilde{B} / B$, is generally low $\left(<10^{-4}\right.$ in tokamaks), it can cause significant transport by short circuiting neighboring flux surfaces, an effect that is proportional to the parallel electron velocity and is thus magnified for LHCD suprathermals. In a variety of studies, based on global power balance $e^{20,32,35,36}$ or on modulation methods, ${ }^{20,37}$ in some cases with the assistance of transport and Fokker-Planck models, ${ }^{20,38,39}$ suprathermal electron transport was found to be negligible com- 
pared to collisional phase-space effects. Typical upper bounds to the diffusion coefficients were determined to be of the order of $0.1-1 \mathrm{~m}^{2} / \mathrm{s},{ }^{20,38,40}$ with the notable isolated exceptions of JET ${ }^{40}$ and TORE SUPRA ${ }^{37}$ measurements suggesting values of $6-10 \mathrm{~m}^{2} / \mathrm{s}$. Under these circumstances, fast electrons are born and die at the same location, and their distribution is therefore a good indicator of the noninductive current profile, an assumption that has been widely used in LHCD scenarios. In the case of fully noninductive discharges, this effectively promotes HXR diagnostics to current-profile diagnostics, also opening the possibility of real-time-control applications. More advanced efforts to bring the power of Fokker-Planck quasilinear simulations - in addition to ray-tracing codes - to bear on the interpretation of experimental data have shown that radial transport may not always be negligible, suggesting more cautious interpretative approaches. ${ }^{41}$ The transport properties of runaway electrons have also been investigated in a number of studies with the aid of HXR emission..$^{30,42}$

On the technological side, the development of solid-state detectors paved the way to more compact HXR setups. Initially, the primary detector materials were $\mathrm{Ge}$ and $\mathrm{Si}$, which owing to a modest bandgap, require liquid-nitrogen cooling in most cases of practical interest in the laboratory. These types of detectors have been employed in a variety of laboratory experiments. ${ }^{17,43-46}$ Both $\mathrm{Ge}$ and $\mathrm{Si}$ detectors have been featured prominently in spacecraft instrumentation, particularly in observations of HXR emitted from the solar corona by flares and coronal mass ejections. ${ }^{47}$ More recent is the development of CdTe and CdZnTe (CZT) crystals as room-temperature HXR detectors. CdTe was first adopted by Peysson and co-workers ${ }^{48,49}$ for a two-camera system on the TORE SUPRA tokamak, also notable for being the first tomographic system of its kind. One of the two cameras was later employed on the tokamak à configuration variable $(\mathrm{TCV}),{ }^{50}$ and additional CdTe and CZT detectors have appeared around the world in the past decade. ${ }^{51-54}$ The work performed on TORE SUPRA in this period, which included the familiar diagnosis of the LH-driven current profile as well as novel studies of the effect of sawteeth on suprathermal electrons, ${ }^{55}$ was also accompanied by efforts at more advanced modeling, particularly on the role of backscattering from the material walls in the interpretation of HXR measurements. ${ }^{56}$ Data interpretation was aided by fitting the measurements with the calculated HXR emission from e.d.f.s simulated by Fokker-Planck codes, with free parameters typically appearing in the prescription for cross-field transport. ${ }^{22,57,58}$

This period coincided also with the coming of age of electron-cyclotron heating (ECH) and current drive (ECCD). Because of the different regions of phase space with which ECH interacts, simulations show that the accelerated electrons remain generally more collisional than in the case of LHCD, resulting in weaker HXR emission. This was confirmed in early measurements, which also showed that more suprathermal emission is observed in the case of ECCD, ${ }^{43,44}$ which targets electrons with high parallel velocity, than with pure ECH. These measurements, particularly on the $T-10$ tokamak, were used to study the decay time upon power

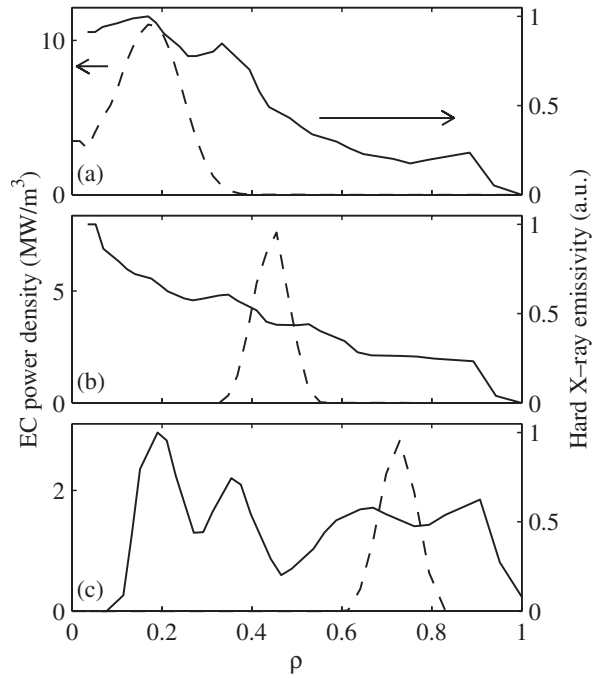

FIG. 4. Local HXR (40-50 keV) emissivity reconstructed from lineintegrated measurements on the TCV tokamak (solid lines) for three different ECCD power deposition profiles (dashed lines), calculated by ray tracing. Reprinted with permission from Coda et al. (Ref. 58).

switch-off, ${ }^{43}$ again concluding that cross-field transport was negligible. ${ }^{59}$ This conclusion was challenged when measurements were performed with ECCD on TCV, which features extremely high (up to $40 \mathrm{MW} / \mathrm{m}^{3}$ ) ECH power densities. In this device, radial transport was clearly dominant over collisional slowing down, as proven by the steady-state HXR emission profiles being considerably broader than the theoretical power deposition profiles ${ }^{58}$ (see Fig. 4). This was attributed to the high power density itself, acting both to accelerate electrons to higher energies (and thus longer collisional lifetimes) and to potentially increase the underlying turbulence that is at the root of anomalous transport. Transport was observed directly by analyzing the evolution of the HXR spectra in space following ECH turn-on, and was estimated to be of the order of $3 \mathrm{~m}^{2} / \mathrm{s}$ in typical scenarios. ${ }^{58}$

Additional studies were performed on TCV on simultaneous low-field-side (LFS) second (X2) and third (X3) harmonic $X$-mode $\mathrm{ECH}$, which resulted in anomalously high (up to $100 \%) X 3$ absorption. A strong dependence of the absorbed power fraction on the parallel wave number of the $X 2$ wave was observed, mirrored in a qualitatively similar dependence of the total HXR emissivity, suggesting that the anomalous $X 3$ absorption was mediated by the suprathermal electrons generated by $X 2{ }^{60}$

An area of growing interest is the application of HXR measurements to the investigation of streaming suprathermal electrons accelerated along the field lines by electric fields generated in magnetohydrodynamics (MHD) events, particularly magnetic-reconnection events. A number of earlier studies focused on runaway production. ${ }^{61,62}$ In more recent years, Savrukhin and co-workers ${ }^{63-65}$ on the $T$ -10 tokamak have laid a solid foundation for investigations of a broad range of MHD phenomena. These studies generally forego energy discrimination in order to maximize the photon statistics and thus the temporal resolution needed to access the fast dynamics of these events, triggered by the sawtooth or the disruptive instability. Multiwire proportional 
chambers ${ }^{66}$ may also be employed for this purpose, although they are primarily sensitive to the soft-x-ray region and do not access the full spectrum of suprathermal emission.

On the hardware front, progress has not stopped at CdTe and CZT detectors, although these remain the state of the art at the time of writing. Alternative higher- $Z$ crystals, while still largely at the experimental stage, are beginning to find their way into practical laboratory applications-a salient example being the $\mathrm{HgI}_{2}$ detectors currently employed on the HL-1M tokamak. ${ }^{67}$

Finally, the development of the first real-time-control applications of HXR measurements deserves mention. On TORE SUPRA, the signal from the HXR camera has been used in real time to vary the parallel wave number of the launched lower-hybrid wave in order to control the plasma's internal inductance. ${ }^{68}$ These are promising, though preliminary, results, belonging to a growing body of real-time algorithms that are likely to dominate the landscape of fusion research for some time to come.

\section{ELECTRON-CYCLOTRON RADIATION}

In magnetized plasmas, cyclotron radiation is emitted owing to the electron gyromotion. ${ }^{2}$ The fundamental angular frequency of this radiation is $\omega=\omega_{\mathrm{ce}} / \gamma+k_{\|} v_{\|}$, where $\omega_{\mathrm{ce}}$ is the angular cyclotron frequency, $\gamma$ is the relativistic energy parameter, $k$ is the radiation wave vector, $v$ is the electron velocity, and the parallel direction is intended relative to the local magnetic field. Emission occurs at all harmonics of this frequency and in both polarizations, the generalized $O$ - and $X$-modes. ${ }^{69}$ In many situations of interest, the plasma acts as a blackbody, and the spatial variation of $\omega_{\text {ce }}$ then allows ECE to be used as a local temperature diagnostic. ${ }^{70}$ With typical laboratory magnetic fields, the frequencies at play are in the microwave range.

However, the velocity dependence of the resonant frequency complicates the picture when suprathermal electrons are present. The fundamental emission angular frequency can be rewritten as $\omega / \omega_{\mathrm{ce}}=\left(1-\beta^{2}\right)^{1 / 2} /\left(1-\beta_{\|} \cos \theta\right)$, where $\beta$ is the relativistic normalized velocity and $\theta$ is the angle of the wave vector to the magnetic field. Contour plots of this function for two values of $\theta$ are shown in Fig. 5. High perpendicular velocities cause a relativistic frequency downshift (or for a given frequency, a shift to higher magnetic field), and high forward parallel velocities (up to a certain limit) cause a Doppler upshift (or a shift to lower field) in the case $k_{\|} \neq 0$. Thus, ECE detection from the high-field side (HFS) of the resonance is a sensitive diagnostic of high- $v_{\perp}$ electrons whereas oblique LFS detection targets preferentially high- $v_{\|}$ electrons. ${ }^{71,72}$ The photon statistics of cyclotron emission being generally far better than those of HXR bremsstrahlung, exploiting ECE for diagnosing suprathermal electrons offers in principle a way to achieve superior time resolution. On the other hand, ECE suffers from the significant complication that the energy and the spatial location of the emitting electrons cannot be determined independently in general.

Historically, the relativistic downshift received the earliest attention. Detection on the HFS of toroidal devices (where the main magnetic field is inversely proportional to

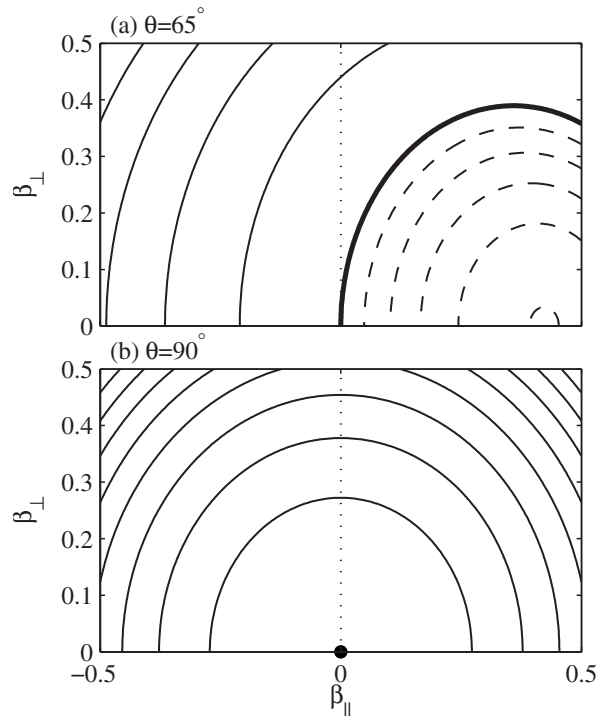

FIG. 5. Contours of emission frequency divided by the cyclotron frequency as functions of the normalized electron velocities parallel and perpendicular to the magnetic field, for (a) oblique emission and (b) perpendicular emission: downshifted $\left(\omega / \omega_{\mathrm{ce}}<1\right.$, solid), $\omega / \omega_{\mathrm{ce}}=1$ (thick), and upshifted $\left(\omega / \omega_{\text {ce }}>1\right.$, dashed curves $)$.

the major radius) was initially proposed in a vertically viewing geometry, ${ }^{71,73}$ and this has remained the most common approach, from tokamaks ${ }^{74-76}$ to stellarators. ${ }^{77}$ In this case emission occurs along a direction of constant magnetic field, resulting in a one-to-one correspondence between major radius (or equivalently, frequency) and energy. This comes at the expense of spatial resolution since the emitting body here is a tenuous and thus generally optically thin population of suprathermal electrons, and radiation is accordingly collected from a finite length along the viewing chord. Furthermore, this consideration mandates that a suitable viewing dump, or a retroreflector, be placed on the material surface opposite the viewing antenna in order to prevent radiation emitted by thermal electrons at larger radii from entering the field of view through spurious reflections (see Fig. 6).

Standard microwave detection equipment is used in these applications, including Michelson interferometers with InSb detectors ${ }^{13,78}$ and heterodyne radiometers. ${ }^{74-76}$ To cir-

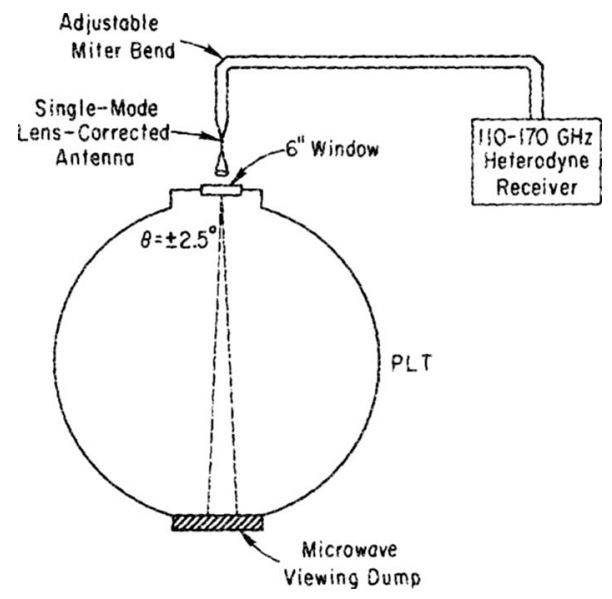

FIG. 6. Schematic of the vertical ECE diagnostic on the PLT tokamak. Reprinted with permission from Luce et al. (Ref. 74). 
cumvent the difficulty of nonlocality, different data-reduction techniques have been employed depending on the physics being targeted. Multiple harmonics have been used in some cases $^{78}$ because of the additional information contained in their different velocity dependences (the emission intensity being proportional to $v^{2 n}$, where $n$ is the harmonic number), and the two polarizations have been compared ${ }^{74}$ to exploit their different dependences on the velocity direction. These techniques permitted the derivation of the average pitch angle of the distribution during LHCD. Direct data comparison with theory-based calculated ECE spectra was also used to constrain the analysis. In this approach a Fokker-Planck simulation is performed to derive the form of the e.d.f., and ECE from this e.d.f. is then calculated and propagated to the antenna by a ray-tracing code. ${ }^{79}$ The question of cross-field transport of ECH-generated suprathermal electrons was addressed by one such study, which found that a satisfactory match was only obtained if transport was included, and that magnetic-turbulence-induced diffusion was more successful than its electrostatic counterpart in explaining the observations. ${ }^{80}$

In a horizontally viewing arrangement on the HFS, energy and emission locations are inextricably tied: the chosen frequency defines the product $\gamma R$ but not the two quantities separately. Radiation will therefore be collected from a region approximately one optical thickness deep as seen from the receiver. Interpretation of these measurements has had to rely on simplified models, most typically a bi-Maxwellian model describing the bulk and suprathermal populations with two different temperatures and densities. ${ }^{81}$ In experiments with low-duty-cycle ECCD modulation accompanied by coherent averaging, this approach has permitted the estimation of the suprathermal density and the direct visualization of the temporal dynamics of the pulse being transported in space from the power deposition region. ${ }^{82}$ Fokker-Planck models have also been used to simultaneously fit LFS and HFS measurements, with suprathermal electron transport supplying the free parameters. ${ }^{83}$ A similar comparative approach using ad hoc e.d.f. models has been applied to the study of MHDmediated suprathermal electron acceleration in conjunction with sawtooth crashes in a tokamak. ${ }^{84}$

The oblique LFS detection geometry has also been exploited in the past decade in a handful of experiments. ${ }^{85-89}$ The nature of the relativistic resonance condition is such that for a given frequency and $k_{\|}$there is a maximum major radius from which radiation can be emitted in a toroidal device (corresponding to the maximum value of $\omega / \omega_{\mathrm{ce}}$ seen near the right-hand boundary of Fig. 5). On the other hand, the oblique detection intrinsically defines a minimum radius, the radius of tangency to the local flux surface. By a judicious choice of parameters, the minimum and maximum radii can be made to be close, resulting in a localized measurement in both space and energy. This approach was exploited with some success on the PBX-M tokamak to study LHCDgenerated suprathermal distributions. ${ }^{90}$ An elegant experiment was recently performed on TCV, comparing co- and counter-ECCD as well as co- and counter-ECE views to directly visualize the ECCD-induced e.d.f. asymmetry. ${ }^{89}$

The most traditional observation geometry for thermal
ECE uses perpendicular LFS views. With $k_{\|}$strictly zero, a LFS view of an optically thick plasma will be unaffected by a non-Maxwellian suprathermal tail. However, the occurrence of optically thin phases in a discharge, as well as the finite $k_{\|}$spectrum admitted by any real antenna, result in possible signal "pollution" in the presence of suprathermal electrons. ${ }^{91}$ It should be noted that the other standard temperature diagnostic technique in high-temperature plasmas, Thomson scattering, is also perturbed by nonthermal electrons in a manner defined by the instrumental spectral response function. ${ }^{92,93}$ It has recently been proposed that the discrepancies between ECE and Thomson-scattering measurements could themselves be exploited to diagnose the suprathermal population, ${ }^{94}$ although the approach appears difficult and has not seen any concrete applications to date. Additionally, with an opportune choice of parameters and harmonic number it is possible to detect ECE from the LFS when the resonance for electrons at rest is located behind the antenna and outside the plasma: the radiation detected can then only be due to downshifted emission from high-energy electrons. In the appropriate conditions this geometry may allow a deconvolution of energy and spatial location. ${ }^{95}$

A rather extreme example of ECE occurs in the presence of strongly relativistic, multi-MeV populations of runaway electrons, for which the radiation-here more appropriately termed synchrotron radiation-is emitted predominantly in the infrared range. Infrared imaging instruments may then be used to diagnose the runaway beams. ${ }^{96}$

Čerenkov emission is also a related form of radiation that can be generated by relativistic particles. In an unmagnetized plasma, this radiation takes the form of a longitudinal electrostatic wave that can, in a number of laboratory situations, be converted into an electromagnetic wave and escape the plasma. Although practical applications have been rare, this radiation has been observed and used to deduce the presence of runaway electrons. ${ }^{97}$

The reciprocal process of electron-cyclotron absorption (ECA) can also be used in addition to ECE, having the practical advantage of being largely unaffected by multiple reflections. For practical reasons, this type of active measurement has been proven most suitable for an oblique LFS geometry in which the probing beam is launched almost vertically in a toroidal system, with only a small $k_{\|}$ component. ${ }^{98}$ With an appropriate choice of parameters, a nearly one-to-one correspondence between major radius (or frequency) and parallel velocity can be achieved, surrendering spatial resolution. ${ }^{99}$ In particular, information on the average skewness of the parallel distribution can be obtained readily by comparing similar measurements with opposite $k_{\|}$, in which all effects attributable to the bulk plasma, such as refraction and scattering, are equal. This type of setup, illustrated by Fig. 7, is naturally attractive for the investigation of LHCD plasmas, and most of the implementations have accordingly been in conjunction with LHCD experiments. ${ }^{99-106}$

A variant of this approach consists of launching the waves from the LFS in an oblique, approximately horizontal direction, and measuring the power reflected back from a cutoff layer. The relative attenuation is then attributed to ECA by resonant electrons situated on the LFS of the cutoff, 


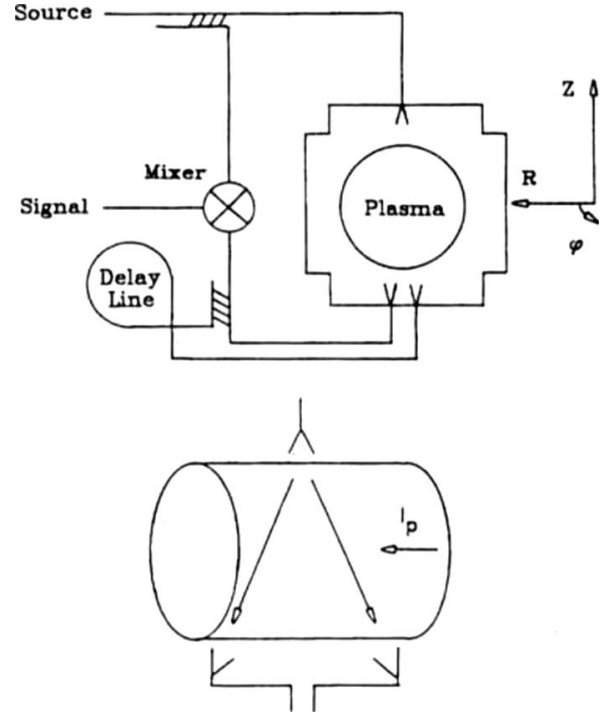

FIG. 7. Experimental configuration of a vertical two-ray ECA diagnostic. Reprinted with permission from Kirkwood et al. (Ref. 99).

which for appropriate choices of parameters can be selected to be suprathermal within a particular range of velocities. ${ }^{107}$

\section{OTHER MEASUREMENT TECHNIQUES}

A variety of additional measurement possibilities emerge when we allow the plasma to be at sufficiently low temperature for being accessed by material probes or to contain heavy enough elements for atomic line radiation to be emitted.

Atomic spectroscopy is employed widely in plasma physics and is of great value whenever partially ionized atoms are present. From light to heavy elements, line emission can be used to diagnose plasma properties from $\mathrm{eV}$ well into $\mathrm{keV}$-temperature plasmas. The relatively recent technique of plasma polarization spectroscopy is based on the "memory" that excited atoms retain of the velocity distribution of the electrons that caused the excitation. The polarization of light emitted by these excited atoms can then be used to extract information on the e.d.f. ${ }^{108-110}$ Applications to laser plasmas $^{111}$ and to tokamak plasmas ${ }^{112}$ have been limited thus far.

Langmuir probes, a standard and time-honored diagnostic of low-temperature plasmas and in universal use at the edge of high-temperature fusion devices, can also be employed to extract the details of the e.d.f., ${ }^{70}$ most commonly by calculating the first or the second derivative of the probe current. While applications to low-temperature laboratory and space plasmas are common, ${ }^{113,114}$ this technique has enjoyed limited interest in fusion experiments. ${ }^{115}$

In situ electron spectrometers are in some way the ultimate tool to measure the e.d.f. where environmental conditions permit it. This is the case in space, where electrostatic analyzers, electron multipliers (channeltrons and microchannel plates), and Si detectors with thin entrance contacts ${ }^{116}$ are employed on a variety of spacecraft, and in basic plasmaphysics laboratory experiments. Again, this type of instrumentation can also be used in some cases in the edge region of fusion devices, as in the case of the pinhole electrostatic analyzer adopted on the Madison symmetric torus (MST) spherical tokamak. ${ }^{117}$

Finally, a few words must be said on indirect measurements that do not probe the suprathermal electrons directly but can be used to reveal their presence. An important example is radio waves from the solar corona. Radio bursts, particularly of type III, are generated by conversion from plasma waves, which are driven by unstable, bump-on-tail nonthermal distributions. Measurements of the energy flux attributed to suprathermal electrons are also performed by detecting the resulting light emission, in the infrared to ultraviolet range: for instance, white-light imaging has been widely employed in the investigation of the corona, and UV spectrometers are used to probe the nonthermal electrons in the terrestrial aurora. ${ }^{118}$ In the laboratory, pyrobolometers ${ }^{119}$ and optical pyrometers ${ }^{27}$ have been in use for decades, particularly in laser fusion.

\section{ACKNOWLEDGMENTS}

Dr. B. P. Duval is gratefully acknowledged for his critical reading of the manuscript. This work was supported in part by the Swiss National Science Foundation.

${ }^{1}$ J. D. Jackson, Classical Electrodynamics (Wiley, New York, 1998).

${ }^{2}$ G. Bekefi, Radiation Processes in Plasmas (Wiley, New York, 1966).

${ }^{3}$ S. von Goeler et al., Diagnostics for Fusion Reactor Conditions, Proceedings of the International School of Plasma Physics, Varenna (Pergamon, London, 1982), Vol. I, p. 87.

${ }^{4}$ J. L. Shohet, Phys. Fluids 11, 1065 (1968).

${ }^{5}$ S. von Goeler and W. Stodiek, Proceedings of the 5th EPS Conference on Plasma Physics and Controlled Fusion, Grenoble, 1972 (European Physical Society, Petit-Lancy, 1972), Vol. 1, p. 2.

${ }^{6}$ S. von Goeler et al., Nucl. Fusion 25, 1515 (1985).

${ }^{7}$ R. Kaita et al., Rev. Sci. Instrum. 61, 2756 (1990).

${ }^{8}$ S. Bernabei et al., Phys. Rev. Lett. 49, 1255 (1982).

${ }^{9}$ M. J. Mayberry et al., Phys. Rev. Lett. 55, 829 (1985).

${ }^{10}$ M. Brambilla, Kinetic Theory of Plasma Waves: Homogeneous Plasmas (Oxford University Press, Oxford, 1998).

${ }^{11}$ J. Stevens et al., Nucl. Fusion 25, 1529 (1985).

${ }^{12}$ S. Texter et al., Nucl. Fusion 26, 1279 (1986).

${ }^{13}$ I. H. Hutchinson, K. Kato, and S. C. Texter, Rev. Sci. Instrum. 57, 1951 (1986).

${ }^{14}$ K. Ogura et al., Nucl. Fusion 31, 1015 (1991).

${ }^{15}$ J. P. Squire et al., Phys. Rev. E 50, 513 (1994).

${ }^{16}$ M. Brusati et al., Nucl. Fusion 34, 23 (1994).

${ }^{17}$ H. Kawashima et al., Jpn. J. Appl. Phys., Part 1 33, 3590 (1994).

${ }^{18}$ S. von Goeler et al., Phys. Plasmas 2, 205 (1995).

${ }^{19}$ R. E. Bell, Rev. Sci. Instrum. 66, 558 (1995).

${ }^{20}$ R. Bartiromo et al., Nucl. Fusion 33, 1483 (1993).

${ }^{21}$ T. Maehara et al., Phys. Lett. A 208, 143 (1995).

${ }^{22}$ J. P. Bizarro et al., Phys. Fluids B 5, 3276 (1993).

${ }^{23}$ S. von Goeler et al., Rev. Sci. Instrum. 65, 1621 (1994).

${ }^{24}$ S. von Goeler et al., Fusion Eng. Des. 34-35, 97 (1997).

${ }^{25}$ D. W. Phillion et al., Phys. Rev. Lett. 49, 1405 (1982).

${ }^{26}$ C. Stoeckl et al., Rev. Sci. Instrum. 72, 1197 (2001).

${ }^{27}$ H. G. Ahlstrom, Appl. Opt. 20, 1902 (1981).

${ }^{28}$ J. D. Strachan, Nucl. Fusion 16, 743 (1976).

${ }^{29}$ S. J. Zweben, D. W. Swain, and H. H. Fleischmann, Nucl. Fusion 18, 1679 (1978).

${ }^{30}$ O. J. Kwon et al., Nucl. Fusion 28, 1931 (1988).

${ }^{31}$ D. Ress, B. W. Rice, and R. D. Horton, Rev. Sci. Instrum. 61, 2777 (1990).

${ }^{32}$ K. Ushigusa et al., Nucl. Fusion 32, 1977 (1992).

${ }^{33}$ P. Martin et al., Rev. Sci. Instrum. 66, 431 (1995).

${ }^{34}$ S. C. Luckhardt, Nucl. Fusion 27, 1914 (1987).

${ }^{35}$ S. Knowlton et al., Phys. Rev. Lett. 57, 587 (1986).

${ }^{36}$ S. Texter et al., Phys. Lett. A 175, 428 (1993).

${ }^{37}$ D. Moreau and C. Gormezano, Plasma Phys. Controlled Fusion 33, 1621 
(1991).

${ }^{38}$ S. Jones et al., Plasma Phys. Controlled Fusion 35, 1003 (1993).

${ }^{39}$ S. E. Jones et al., Phys. Plasmas 2, 1548 (1995).

${ }^{40}$ Y. Peysson, Plasma Phys. Controlled Fusion 35, B253 (1993).

${ }^{41}$ G. Giruzzi, Phys. Fluids B 4, 1391 (1992).

${ }^{42}$ P. J. Catto et al., Phys. Fluids B 3, 2038 (1991).

${ }^{43}$ V. V. Alikaev et al., Nucl. Fusion 32, 1811 (1992).

${ }^{44}$ D. F. da Cruz, Jr., J. H. Meijer, and A. J. H. Donné, Rev. Sci. Instrum. 63, 5026 (1992)

${ }^{45}$ S. Muto et al., Rev. Sci. Instrum. 74, 1993 (2003).

${ }^{46}$ T. I. Madeira et al., Rev. Sci. Instrum. 78, 086108 (2007).

${ }^{47}$ R. P. Lin et al., Sol. Phys. 210, 3 (2002).

${ }^{48}$ Y. Peysson and R. Arslanbekov, Nucl. Instrum. Methods Phys. Res. A 380, 423 (1996).

${ }^{49}$ Y. Peysson and F. Imbeaux, Rev. Sci. Instrum. 70, 3987 (1999).

${ }^{50}$ Y. Peysson, S. Coda, and F. Imbeaux, Nucl. Instrum. Methods Phys. Res. A 458, 269 (2001)

${ }^{51}$ R. O'Connell et al., Rev. Sci. Instrum. 74, 2001 (2003).

${ }^{52}$ D. T. Anderson et al., Fusion Sci. Technol. 50, 171 (2006).

${ }^{53}$ P. K. Sharma et al., Fusion Eng. Des. 82, 41 (2007).

${ }^{54} \mathrm{~S}$. Gnesin et al., Rev. Sci. Instrum. (to be published).

${ }^{55}$ F. Imbeaux and Y. Peysson, Phys. Rev. Lett. 84, 2873 (2000).

${ }^{56}$ Y. Peysson, P. Froissard, and C. Pocheau, Nucl. Fusion 33, 1133 (1993).

${ }^{57}$ P. Nikkola et al., Nucl. Fusion 43, 1343 (2003)

${ }^{58}$ S. Coda et al., Nucl. Fusion 43, 1361 (2003).

${ }^{59}$ Yu. V. Esipchuk et al., Fiz. Plazmy 21, 577 (1995)[Plasma Phys. Rep. 21, 577 (1995)].

${ }^{60}$ S. Alberti et al., Nucl. Fusion 42, 42 (2002).

${ }^{61}$ C. W. Barnes et al., Phys. Lett. 81A, 275 (1981).

${ }^{62}$ R. D. Gill et al., Nucl. Fusion 40, 163 (2000).

${ }^{63}$ P. V. Savrukhin and I. V. Klimanov, Rev. Sci. Instrum. 72, 1668 (2001).

${ }^{64}$ P. V. Savrukhin, Phys. Rev. Lett. 86, 3036 (2001).

${ }^{65}$ P. V. Savrukhin, Plasma Phys. Controlled Fusion 48, B201 (2006).

${ }^{66}$ A. Sushkov et al., Rev. Sci. Instrum. 79, 023506 (2008).

${ }^{67}$ Y. Jinwei, Z. Wei, and W. Shiqing, Rev. Sci. Instrum. 75, 2513 (2004).

${ }^{68}$ O. Barana et al., Plasma Phys. Controlled Fusion 49, 947 (2007).

${ }^{69}$ M. Bornatici et al., Nucl. Fusion 23, 1153 (1983).

${ }^{70}$ I. H. Hutchinson, Principles of Plasma Diagnostics (Cambridge University Press, Cambridge, England, 2005).

${ }^{71}$ C. M. Celata and D. A. Boyd, Nucl. Fusion 17, 735 (1977).

${ }^{72}$ S. Tamor, Nucl. Fusion 19, 455 (1979).

${ }^{73}$ I. H. Hutchinson and K. Kato, Nucl. Fusion 26, 179 (1986).

${ }^{74}$ T. C. Luce, P. C. Efthimion, and N. J. Fisch, Rev. Sci. Instrum. 59, 1593 (1988).

${ }^{75}$ S. Ide et al., Nucl. Fusion 29, 1325 (1989).

${ }^{76}$ D. R. Roberts et al., Rev. Sci. Instrum. 66, 427 (1995).

${ }^{77}$ W. Pernreiter et al., Proceedings of the Tenth Joint Workshop on Electron Cyclotron Emission and Electron Cyclotron Resonance Heating, Ameland, 1997 (World Scientific, Singapore, 1998), p. 241.

${ }^{78}$ K. Kato and I. H. Hutchinson, Phys. Rev. Lett. 56, 340 (1986)

${ }^{79}$ G. Giruzzi, Plasma Phys. Controlled Fusion 35, 1541 (1993).

${ }^{80}$ G. Giruzzi et al., Plasma Phys. Controlled Fusion 38, 1593 (1996).

${ }^{81}$ P. Blanchard et al., Plasma Phys. Controlled Fusion 44, 2231 (2002).

${ }^{82}$ S. Coda et al., Plasma Phys. Controlled Fusion 48, B359 (2006).
${ }^{83}$ J. F. M. van Gelder et al., Plasma Phys. Controlled Fusion 40, 1185 (1998).

${ }^{84}$ I. Klimanov et al., Plasma Phys. Controlled Fusion 49, L1 (2007).

${ }^{85}$ L. Rodriguez et al., Proceedings of the 18th EPS Conference on Plasma Physics and Controlled Fusion, Berlin, 1991 (European Physical Society, Mulhouse, 1991), Vol. 15C, p. IV-353.

${ }^{86}$ S. Preische, P. C. Efthimion, and S. M. Kaye, Phys. Plasmas 3, 4065 (1996).

${ }^{87}$ E. de la Luna et al., Proceedings of the 12th Joint Workshop on Electron Cyclotron Emission and Electron Cyclotron Heating (EC-12), Aix-enProvence, 2002 (World Scientific, Singapore, 2003), p. 177.

${ }^{88}$ C. Sozzi et al., Fusion Eng. Des. 74, 691 (2005).

${ }^{89}$ T. P. Goodman et al., Proceedings of the 34th EPS Conference on Plasma Physics and Controlled Fusion, Warsaw, 2007 (European Physical Society, Mulhouse, 2007) [Europhys. Conf. Abstr. 31F, 2.147 (2007)].

${ }^{90}$ S. Preische, P. C. Efthimion, and S. M. Kaye, Rev. Sci. Instrum. 68, 409 (1997).

${ }^{91}$ H. Kawashima et al., Nucl. Fusion 31, 495 (1991)

${ }^{92}$ E. de la Luna et al., Rev. Sci. Instrum. 74, 1414 (2003).

${ }^{93}$ G. Zhuang et al., Plasma Phys. Controlled Fusion 47, 1539 (2005).

${ }^{94}$ V. Krivenski, Fusion Eng. Des. 53, 23 (2001).

${ }^{95}$ B. C. Schokker et al., Plasma Phys. Controlled Fusion 37, 1299 (1995).

${ }^{96}$ I. Entrop et al., Plasma Phys. Controlled Fusion 40, 1513 (1998).

${ }^{97}$ I. H. Hutchinson and D. S. Komm, Nucl. Fusion 17, 1077 (1977).

${ }^{98}$ E. Mazzucato, P. Efthimion, and I. Fidone, Nucl. Fusion 25, 1681 (1985).

${ }^{99}$ R. K. Kirkwood et al., Nucl. Fusion 30, 431 (1990).

${ }^{100}$ R. K. Kirkwood et al., Phys. Fluids B 2, 1421 (1990).

${ }^{101}$ R.K. Kirkwood et al., Nucl. Fusion 31, 1938 (1991).

${ }^{102}$ T. Dudok de Wit et al., Proceedings of the 20th EPS Conference on Plasma Physics and Controlled Fusion, Lisboa, 1993 (European Physical Society, Petit-Lancy, 1993), Vol. 17C, p. III-1095.

${ }^{103}$ F. Skiff, D. A. Boyd, and J. A. Colborn, Phys. Fluids B 5, 2445 (1993).

${ }^{104}$ J. L. Ségui and G. Giruzzi, Plasma Phys. Controlled Fusion 36, 897 (1994).

${ }^{105}$ G. Giruzzi et al., Phys. Rev. Lett. 74, 550 (1995).

${ }^{106}$ D. A. Boyd, F. Skiff, and S. Gulick, Rev. Sci. Instrum. 68, 496 (1997).

${ }^{107}$ G. Giruzzi and I. Fidone, Plasma Phys. Controlled Fusion 33, 1243 (1991).

${ }^{108}$ R. Fischer and V. Dose, Plasma Phys. Controlled Fusion 41, 1109 (1999).

${ }^{109}$ A. Iwamae et al., Plasma Phys. Controlled Fusion 47, L41 (2005).

${ }^{110}$ T. Fujimoto and S. A. Kazantsev, Plasma Phys. Controlled Fusion 39, 1267 (1997).

${ }^{111}$ J. C. Kieffer et al., Phys. Rev. Lett. 68, 480 (1992).

${ }^{112}$ T. Fujimoto et al., Phys. Rev. E 54, R2240 (1996).

${ }^{113}$ V. A. Godyak, Plasma-Surface Interaction and Processing of Materials (Kluwer, Deventer, 1990), p. 95.

${ }^{114}$ V. I. Demidov, S. V. Ratynskaia, and K. Rypdal, Rev. Sci. Instrum. 73, 3409 (2002).

${ }^{115}$ K. Uehara et al., Jpn. J. Appl. Phys., Part 1 42, 657 (2003).

${ }^{116}$ C. S. Tindall et al., IEEE Trans. Nucl. Sci. 55, 797 (2008).

${ }^{117}$ T. D. Rempel et al., Phys. Fluids B 4, 2136 (1992).

${ }^{118}$ J.-C. Gérard et al., Planet. Space Sci. 56, 542 (2008).

${ }^{119}$ G. Fiksel, J. Frank, and D. Holly, Rev. Sci. Instrum. 64, 2761 (1993). 